\title{
THE RELATIONSHIP BETWEEN PROLACTIN LEVELS AND THE RESULTS OF HOLTER MONITORING IN PATIENTS WITH STABLE ANGINA OF TENSION AND GASTROESOPHAGEAL REFLUX DISEASE
}

D0I: 10.36740/WLek202003118

\author{
Oksana S. Khukhlina, Alona A. Antoniv, Viktoriia Yu. Drozd, Zoriana la. Kotsiubiichuk, Vitaliy S. Smandych \\ HIGHER STATE EDUCATIONAL INSTITUTION OF UKRAINE"BUKOVINIAN STATE MEDICAL UNIVERSITY", CHERNIVTSI, UKRAINE
}

\begin{abstract}
The aim: Determine the possible relationship between prolactin levels and the results of Holter monitoring in patients with stable angina of tension and gastroesophageal reflux disease (GERD).

Materials and methods: The study included 118 patients with stable angina of tension of $I-I$ f functional class. Of these, 88 patients with stable angina of tension with comorbid GERD $(A, B, C)$, who were included in the second group of the study and 30 patients with isolated stable angina of tension which formed the 1st group. All patients who were included in the study prior to treatment were evaluated for serum prolactin levels and Holter monitoring.

Results: In patients with stable angina of tension and GERD, prolactin levels exceed the norm levels and are higher than the level of prolactin in the group with isolated stable angina $(p<0,05)$, as well as the number of episodes of painful and painless myocardial ischemia and their duration $(p<0,05)$. The presence of a comorbidity with GERD provokes an increase in the total duration of episodes of ischemia per day in patients with stable angina compared to those in patients with no GERD. A direct linear relationship was founded between prolactin serum levels and the number of episodes of pain in myocardial ischemia in patients with stable angina and GERD per day, and between the total duration of episodes of myocardial ischemia and prolactin levels.

Conclusions: In patients with stable angina and GERD, in $70.4 \%$ of cases, the level of prolactin exceeds the norm in 2,6 times, and is higher than the level of prolactin in the isolated group of stable angina. For the comorbidity of stable angina and GERD, the number of episodes of painful, painless myocardial ischemia and their duration is more than once in the group of patients with stable angina without GERD $(p<0.05)$.
\end{abstract}

KEY WORDS: Stable angina of tension, gastroesophageal reflux disease, prolactin, Holter monitoring, stress

Wiad Lek. 2020;73(3):504-507

\section{INTRODUCTION}

It is known that stress plays a role in the pathophysiology of chronic diseases of the gastrointestinal tract, the cardiovascular system and the endocrine system. Psychological stress is one of the factors of the onset and progression of myocardial lesions $[1,2,3]$. Stress is a critical factor that leads to the development of depressive disorders and hypersensitization. The influence on the development of pathological processes in the human body of chronic stress is also established. According to the conducted meta-analysis, chronic stress was found not to be a risk factor for chronic myocardial ischemia only, but also provokes the appearance of acute cardiovascular events in patients with coronary heart disease [4], causing persistent endothelial dysfunction [4]. One of the main intermediaries and indicators of both acute and chronic stress is prolactin hormone [3,4-6,7]. It is excreted in combination with other pituitary hormones in response to the influence of stress factors and performs the tread effect by changing neuronal co-operation. However, long-term increase in the level of prolactin in the bloodstream may lead to the development of pathological changes caused by hyperprolactinemia. Thus, it is reported that there is a close relationship between elevated levels of prolactin and psychological derivations in the form of the causing or increasing anxiety and irritability $[7,8]$. The level of prolactin above the norm promotes the development of depressive behavior [8]. Against the background of elevated levels of prolactin, the level of the vasoingibin hormone increases in the blood, which is a proteolytically cleaved prolactin that loses the ability to interact with prolactin receptors, but inhibits angiogenesis, neovascularization, narrowing lumen and vascular permeability, contributing to the onset or progression of endothelial dysfunction [8-10] Under conditions of chronic stress, elevated levels of prolactin stimulate the functional activity of fibroblasts in the myocardium, on the surface of which the receptor is expressed in the prolactin [6], which leads to the occurrence of cardiosclerosis and arrhythmias [10]. At the same time carrying out of the medical correction of hyperprolactinemia correlates with decrease in 
proliferation of fibroblasts [6]. An elevated prolactin level inhibits NO-mediated vasodilation of coronary arteries due to the activation of $\beta 2$-adrenergic receptors in them $[11,12-14]$. The inhibitory effect of hyperprolactinemia on proliferation and regenerative properties of the epithelium of the gastrointestinal tract has been established [15]. In addition, chronic stress provokes the persistence of hyperalgesia in inflamed areas of the gastrointestinal tract $[4,15]$, which can exacerbate the manifestations of somatic pain as it stimulates perception and a sense of physical pain and discomfort behind the sternum. According to the literature analysis, the growth of prolactin levels in the range of 25$80 \mathrm{ng} / \mathrm{ml}$ is a consequence of stress induction. The level of prolactin increases in the human body under the influence of certain stress factors, so it can be considered an indicator of "chronic stress" $[10,16]$.

\section{THE AIM}

To determine the possible relationship between prolactin levels and the results of Holter ECG monitoring in patients with stable angina and GERD.

\section{MATERIALS AND METHODS}

The study included 118 patients with stable angina pectoris of I-II functional class aged from 48 to 79 years. Of these, 88 patients with stable angina with comorbid GERD (A, B, C by the Los Angeles classification), who were included in the second group of the study and 30 patients with isolated stable angina which formed the 1st group. Diagnosis of stable angina was established on the basis of the Order of the Ministry of Health of Ukraine No. 152 of March 2, 2016 "Stable Ischemic Heart Disease". The diagnosis of GERD was established on the Lyon Consensus [17]. All patients who were included in the study prior to treatment had a determination of serum level of prolactin by immunoassay. Blood collection for determining the level of prolactin was performed in the morning, immediately after waking up from the elbow, in the minimum stressful conditions. The upper limit of the norm of prolactin level was the average value between the upper limit of the norm for men and women, which is $22.5 \mathrm{ng} / \mathrm{ml}$. All women who participated in the study were in the post-menopausal period. With the help of daily ECG Holter monitoring (HM) results were obtained and we analyzed the total duration of myocardial ischemia (min/day), the number of episodes of painful and painless myocardial ischemia per day (n), the mean duration of episodes of painful myocardial ischemia and painless myocardial ischemia ( $\mathrm{min} /$ day) To determine the episodes of myocardial ischemia using the Kodama criteria [2]. Other indicators that have been set up using HM ECG in this article will not be described.

Statistical processing of the obtained results was carried out using the software Microsoft Excel 2013. The distribution of samples was checked for normality using the criterion Shapiro-Wilka. The samples that had a normal distribution were checked using the parametric method, the Student's t-criterion for independent samples.
The authors declare that all the procedures and experiments of this study respect the ethical standards in the Helsinki Declaration of 1975, as revised in 2008(5), as well as the national law. Opinion of the Bioethical Commission on Higher State Educational Institution of Ukraine "Bukovinian State Medical University" of 3 October 2020.

\section{RESULTS}

According to the results of the study, the incidence of hyperprolactinemia in patients included in the 2nd study group was $70.4 \%$, that is in 62 of the 88 patients with stable angina with comorbid gastrointestinal reflux disease, serum prolactin levels were found to be higher than normal. Concerning the frequency of hyperprolactinemia in patients with isolated stable angina, in 17 of 30 patients, an elevated prolactin serum level was detected at 56\%. By analyzing the results of the prolactin serum levels in both groups of the study, it was found that in the case of an isolated course of stable angina, and with the comorbid flow of stable angina with GERD, the mean value of prolactin level in each group exceeded the norm. However, with the combined course of stable angina and GERD, a significantly higher prolactin level was observed than in the first group, which exceeded the norm by 2.6 times $(p<0, \underline{05})$ (Tab I). In patients of the 1st group, the average prolactin level exceeded the norms in 1.6 times $(\mathrm{p}<0.05)$ (Tab I). Comparing the mean value of the prolactin level of both groups, it was found that in the conditions of the combined course of stable angina and GERD is 1.7 times higher than in the case of an isolated course of stable angina, which is a significant statistical difference $(\mathrm{p}<0.05)$ (Tab I).

The transient increase in prolactin levels is a physiological response to a stress factor, but if the factor is constant or repetitive, the level of prolactin may remain for a long time at a high level, because of the depletion of the inhibitory mechanisms that is controlling the levels of prolactin $[10,16]$. In our opinion, an increase in its level in the blood of patients with stable angina is due to the constant presence of patients in conditions such as psychological (awareness of the presence of the disease, the need for constant intake of medication, regular visits to the district therapist and / or cardiologist, the need for constant control of the disease and the fear of the next chest pain and the fear of death), genetic stress, and physical stress (pain behind the sternum). Under the conditions of the comorbidity of stable angina with GERD, the number of both psychological (awareness of the presence of 2 pathologies, disturbing and obsessive thoughts about the possible iatrogenic effect of drugs, the reception of a large number of medications) and physiological stressors increases due to clinical manifestations of the disease (heartburn, acid regurgitation, dysphagia, odynophagia, pain behind the sternum, which can mimic an attack of angina etc.). Actually, all of the above and leads to chronic stress and hyperprolactinemia. According to Holter's ECG monitoring in the 2nd study group, the mean value of the number of episodes of pain in myocardial ischemia exceeded the similar indicator of 
Table I. Levels of prolactin and ECG Holter monitoring in patients of both groups of study $(M \pm m)$

\begin{tabular}{|c|c|c|c|}
\hline Indicator & Unit & $\begin{array}{c}\text { 1st Group } \\
\text { stable angina only }(n=30)\end{array}$ & $\begin{array}{c}\text { 2nd Group stable angina } \\
+ \text { GERD }(n=88)\end{array}$ \\
\hline Prolactin & $\mathrm{ng} / \mathrm{ml}$ & $35,63 \pm 2,92$ & $58,3 \pm 2,42^{*}$ \\
\hline Total duration of MI & $\min /$ day & $25,76 \pm 1,93$ & $34,96 \pm 1,36^{*}$ \\
\hline Number of painful Ml episodes & $\mathrm{n}$ & $2,96 \pm 0,33$ & $4,59 \pm 0,19^{*}$ \\
\hline Number of painless Ml episodes & & $5,61 \pm 0,3$ & $7,75 \pm 0,36^{*}$ \\
\hline Average duration of painful MI & $\min /$ day & $5,25 \pm 0,48$ & $7,03 \pm 0,44^{*}$ \\
\hline Average duration of painless $\mathrm{Ml}$ & & $4,47 \pm 0,85$ & $6,23 \pm 0,47^{*}$ \\
\hline
\end{tabular}

* - the difference is reliable, compared with the results of patients in the first group.
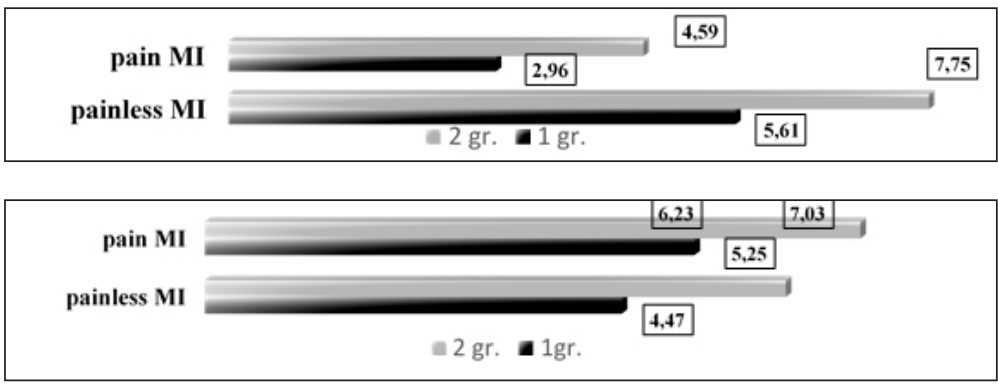

Fig 1.

HM ECG - Holter monitoring of electrocardiography; GERD - gastroesophageal reflux disease

\section{Fig 2.}

HM ECG - Holter monitoring of electrocardiography; GERD - gastroesophageal reflux disease patients with isolated stable angina by 1.5 times $(\mathrm{p}<0.05)$ (Tab I, Fig 1), which can testify to the provoking effect of the inflammatory process of the lower esophagus on the myocardium, under the conditions of the comorbidity of stable angina with GERD. At the same time, the average duration of myocardial ischemia was 1.4 times higher compared with patients in the 1st group (Tab I), (Fig. 2). A similar trend was also observed in the analysis of the average number of episodes of painless myocardial ischemia (Table I, Fig. 1). So the number of episodes in the group of patients with stable angina and GERD exceeded that in the group of patients with isolated stable angina in 1,3 times $(\mathrm{p}<0,05)$ (Fig 1$)$, as well as the mean duration of painless myocardial ischemia in 1,2 times $(\mathrm{p}<0,05)$ (Tab I), (Fig 2).

By conducting an in-depth analysis of the results of the second group patient study, our attention was also drawn to the fact that serum prolactin levels and the number of episodes of pain in myocardial ischemia are depended. It was found that the value of the Pearson correlation coefficient $(r)$ for these indices is $0.34(p<0,05)$, which indicates direct linear relationship between the level of serum prolactin and the number of episodes of pain in myocardial ischemia. The mean interaction correlation $(r=0.25)$ was determined by an analysis between the level of prolactin and the number of episodes of painless myocardial ischemia. Correlation between the duration of pain or painless myocardial ischemia and the prolactin level in patients in group 1 was within the range of weak $(\mathrm{r}=0.15$ and $\mathrm{r}=0.21)(\mathrm{p}<0.05)$, in contrast to the above described correlation in 2nd study group. Thus, in patients with ischemic heart disease and GERD, the highest level of prolactin serum was detected suggested the greater number of episodes of pain in myocardial ischemia was recorded in patients with HM.

\section{DISCUSSION}

In our opinion, the proportional increase in the prolactin level and the number of episodes of pain in the course of the day is due to the presence of patients in conditions of constant stress, which is caused by frequent attacks of remitting pain, which occurs as a result of episodes of myocardial ischemia, and as a result of GERD symptoms. After all, the pain behind the sternum due to gastroesophageal reflux can simulate the clinic of coronary abdominal pain, which leads to a constant fear of a possible occurrence of angina, anxiety and "worst" expectations. Concerning the total duration of episodes of ischemia per day, it was found that this rate of patients in group 2 was 1.4 times that of a group of patients with isolated stable angina (Tab I). This may indicate that the presence of comorbid GERD not only can provoke angina attacks [18], but also affect their duration. We found a correlation relationship $(\mathrm{r}=0.37$, $\mathrm{p}<0.05)$ ) between the total duration of episodes of MI per day and the level of serum prolactin. Consequently, the presence of chronic chest pain due to GERD in patients with stable angina leads to an increase in the level of total MI per day, which is in close correlation with the level of serum prolactin.

\section{CONCLUSIONS}

In patients with stable angina and GERD, in $70.4 \%$ of cases, the level of prolactin exceeds the norm in 2,6 times, and is higher than the level of prolactin in the isolated group of stable angina. For the comorbidity of stable angina and GERD, the number of episodes of painful, painless myocardial ischemia and their duration is more than once in the group of patients with stable angina without GERD $(p<0.05)$. There is a direct linear relationship between 
serum prolactin levels and the number of episodes of painful myocardial ischemia per day in patients with stable angina and GERD. The presence of a comorbid GERD in patients with stable angina leads to an increase in the total duration of episodes of ischemia per day in patients with stable angina compared to patients who have no GERD. We have established correlation between the total duration of episodes of myocardial ischemia and the level of serum prolactin in the blood in patients with stable angina of tension under conditions of comorbid GERD.

\section{REFERENCES}

1. Alevizos M, Karagkouni A, Panagiotidou S et al. Stress triggers coronary mast cells leading to cardiac events. Ann Allergy Asthma Immunol. 2014;112:309-16.

2. Kodama Y. Evalution of myocardial ischemia using Holter monitoring. Fukuoka-Igaku-Zasshi. 995;86(7):304-316.

3. Sztainberg Y, Kuperman Y, Issler 0 et al. A novel corticotropin-releasing factor receptor splice variant exhibits dominant negative activity: a putative link to stress-induced heart disease. FASEB J. 2009;23:2186-96.

4. Wei J, Rooks C, Ramadan R et al. Meta-analysis of mental stress-induced myocardial ischemia and subsequent cardiac events in patients with coronary artery disease. Am J Cardiol. 2014;114:187-92. doi: 10.1016/j. amjcard.2014.04.022

5. Vicario M, Alonso C, Guilarte M et al. Chronic psychosocial stress induces reversible mitochondrial damage and corticotropin-releasing factor receptor type-1 upregulation in the rat intestine and IBS-like gut dysfunction. Psychoneuroendocrinology. 2012;37:65-77. doi: 10.1016/j.psyneuen.2011.05.005

6. Song J, Wang M, Chen $X$ et al. Prolactin mediates effects of chronic psychological stress on induction of fibrofatty cells in the heart. Am J Transl Res. 2016;8(2):644-52.

7. Wu W, Sun M, Zhang HP et al. Prolactin mediates psychological stress-induced dysfunction of regulatory $T$ cells to facilitate intestinal inflammation. Gut. 2014;63:1883-92.

8. Reavley A, Fisher AD, Owen D, Creed FH, Davis JR. Psychological distress in patients with hyperprolactinaemia. Clin Endocrinol (0xf). 1997;47(3):343-8. doi: 10.1046/j.1365-2265.1997.2701073.x

9. Torner L. Actions of Prolactin in the Brain: From Physiological Adaptations to Stress and Neurogenesis to Psychopathology. Front Endocrinol (Lausanne). 2016;30:7:25. doi: 10.3389/fendo.2016.00025.

10. Zamorano M, Ledesma-Colunga MG, Adán N et al. Prolactinderived vasoinhibins increase anxiety-and depression-related behaviors. Psychoneuroendocrinology.2014;44:123-32. doi: 10.1016/j. psyneuen.2014.03.006

11. Ladwig KH, Baumert J, Marten-Mittag B et al. Posttraumatic stress symptoms and predicted mortality in patients with implantable cardioverter-defibrillators: results from the prospective living with an implanted cardioverter-defibrillator study. Arch Gen Psychiatry. 2008;65:1324-30.

12. Gonzalez C, Rosas-Hernandez H, Jurado-Manzano B et al. The prolactin family hormones regulate vascular tone through $\mathrm{NO}$ and prostacyclin production in isolated rat aortic rings. Acta Pharmacol Sin. 2015;36(5):572-86. doi: 10.1038/aps.2014.159.
13. Lafuente A, Gonzalez-Carracedo A, Romero A et al. Effect of nitric oxide on prolactin secretion and hypothalamic biogenic amine contents. Life Sci. 2004;74(13):1681-90.

14. Molinari C, Grossini E, Mary D et al. Prolactin induces regional vasoconstriction through the beta2-adrenergic and nitric oxide mechanisms. Endocrinology. 2007;148:4080-90.

15. Wood S, McFadden K, Grigoriadis D et al. Depressive and cardiovascular disease comorbidity in a rat model of social stress: a putative role for corticotropinreleasing factor. Psychopharmac. (Berl) 2012;222:325-36.

16. Yang P, Jury J, Soderholm J, Sherman P, McKay DM, Perdue MH. Chronic psychological stress in rats induces intestinal sensitization to luminal antigens. Am J Pathol. 2006;168:104-14.

17. Lychkova A.E, Puzikov A.M. Prolaktun i Serotonin [Prolactin and Serotonin]. Vesnik RAMN. 2014;1-2:38-45

18. Gyawali C, Kahrilas P, Savarino E et al. Modern diagnosis of GERD: the Lyon Consensus Gut 2018;67:1351-1362.

19. Tougas G, Spaziani R, Hollerbach S et al. Cardiac autonomic function and oesophageal acid sensitivity in patients with non-cardiac chest pain. Gut. 2001 Nov;49(5):706-712. doi:10.1136/gut.49.5.706

This work is a fragment of the research work "Clinical, pathogenetic and pharmacotherapeutic features of internal diseases», registration number 0119 U101344 (2019-2023).

\section{ORCID and contributionship:}

Oksana S. Khukhlina-0000-0003-2399-512X A, F

Alona A. Antoniv - 000-0003-2399-512X $X^{A, B, C, D}$

Viktoriia Yu. Drozd - 0000-0002-1108-3708 A, B, C, D, E, F

Zoriana Ia. Kotsiubiichuk B, C

Vitaliy S. Smandych - 0000-0002-1959-6575 ${ }^{B, C}$

\section{Conflict of interest:}

The Authors declare no conflict of interest.

\section{CORRESPONDING AUTHOR \\ Alona A. Antoniv \\ Higher Educational Establishment of Ukraine \\ "Bukovinian State Medical University", Teatralnaya Square, 2; 58002, Ukraine, Chernivtsi, tel: 0992321861 \\ e-mail: antonivalona@ukr.net}

Received: 17.01 .2020

Accepted: 05.03 .2020

\footnotetext{
A - Work concept and design, B - Data collection and analysis, C - Responsibility for statistical analysis, D-Writing the article, $\mathbf{E}-$ Critical review, $\mathbf{F}$ - Final approval of the article
} 